Sánchez, M.; García, J.A.; Carcedo, R.; Hernández, D.; Carretero, M. y Sanchez-Sanchez, J. (201x) Is It Decisive the Modality of Competition U-12 in the Dribble of Soccer Players? Revista Internacional de Medicina y Ciencias de la Actividad Física y el Deporte vol. 19 (75) pp. 431-443 Http://cdeporte.rediris.es/revista/revista75/artdeterminante1038.htm

DOI: 10.15366/rimcafd2019.75.004

\title{
ORIGINAL
}

\section{¿ES DETERMINANTE LA MODALIDAD DE COMPETICIÓN EN LA ACCIÓN DE REGATE EN FUTBOLISTAS SUB12?}

\section{IS IT DECISIVE THE MODALITY OF COMPETITION U-12 IN THE DRIBBLE OF SOCCER PLAYERS?}

\author{
Sánchez, M. ${ }^{\text {; }}$ García, J.A. ${ }^{2}$; Carcedo, R. ${ }^{2}$; Hernández, D. ${ }^{3}$; Carretero, M. ${ }^{4}$ y \\ Sanchez-Sanchez, J.4 \\ 1 Universidad Pontificia de Salamanca. Salamanca (España) msanchezga@upsa.es \\ 2 Universidad de Salamanca. Salamanca (España) gherrero@usal.es, rcarcedo@usal.es \\ 3 Preparador Físico de la Federación de Castilla y León de Fútbol. Salamanca (España) \\ danielhdez10@hotmail.com \\ ${ }^{4}$ Grupo de Investigación Planificación y Evaluación del Entrenamiento y Rendimiento \\ Deportivo. Universidad Pontificia de Salamanca. Salamanca (España) mcarreterogo@upsa.es, \\ jsanchezsa@upsa.es
}

Código UNESCO / UNESCO Code: 5899 Otras Especialidades Pedagógicas (Educación Física y Deporte) / Other Educational Specialties (Physical Education and Sport).

Clasificación Consejo de Europa / Classification Council of Europe: 4. Educación Física y deporte comparado/ Physical Education and sport compared.

Recibido 1 de septiembre de 2017 Received September 1, 2017

Aceptado 15 de enero de 2018 Accepted January 15, 2018

\section{RESUMEN}

El objetivo fue analizar la influencia de las modalidades de juego aplicadas a la competición en categoría sub-12 sobre el regate. Se analizaron 18 partidos, 6 por modalidad, registrando 785 regates. Las variables dependientes fueron regates realizados $(R T)$, ganados $(R G)$ y perdidos $(R P)$. Se registraron en función a zonas del campo: pasillos laterales (PLD+PLI) y central (PC); sector defensivo (SD), medio defensivo (SMD), medio ofensivo (SMO) y ofensivo (SO). En fútbol 11 ( $F 11)$ el número de $R G$ fue significativamente mayor $(p<0,05)$ que en Fútbol 7 (F7). Los $R T$ y $R G$ realizados en PLI+PLD fueron mayores $(p<0,05)$ en F11 que F7. Además en fútbol $8(F 8)$ y $F 11$ el RT era mayor $(p<0,01)$ en 
PLD+PLI que en $P C$, también era menor $(p<0,01)$ en SD que en SMD y SO. La dimensión del terreno de juego es determinante en la acción técnica del regate en jugadores de categoría sub-12.

PALABRAS CLAVE: fútbol 7, fútbol 8, fútbol 11, jugador sub12, técnica, regate.

\begin{abstract}
The aim was to analyse the influence of the modalities of the game applied against the competition in the beginner category focusing on the dribble. We analyzed 18 matches, 6 by modality, registering 785 dribbles. The dependent variables were carried out (RT), earned (RG) and lost (RP). They were registered according to areas of the field: lateral corridors (PLD + PLI) and central (PC); defensive (SD), defensive medium (SMD), offensive medium (SMO) and offensive (SO). In Football 11 (F11) the number of RG was significantly higher $(p<0.05)$ than in Football $(F 7)$. The RT and RG performed in $\mathrm{PLI}+\mathrm{PLD}$ in F11 were significantly higher $(p<0.05)$ than in F7. The intramodality analysis showed that in Football (F8) and F11, RT was significantly higher $(p<0.01)$ in PLD + PLI than in PC, and there was lower ( $p$ $<0.01)$ in SD than in SMD and SO. The size of the pitch is decisive in the technical action of dribbling in players of Us12 category.
\end{abstract}

KEY WORDS: football 7, football 8, football 11, player U12, technique, dribble.

\title{
1 INTRODUCCIÓN
}

El rendimiento del futbolista es producto de la interacción de múltiples variables (Reilly, Bangsbo \& Franks, 2000). Algunos estudios han indicado que el éxito en la competición depende de una recíproca combinación de capacidades físicas, facultades perceptivo-decisionales y la habilidad para ejecutar acciones técnicas en un entorno altamente cambiante (Lee, Whitehead \& Ntoumanis, 2007; Nevill, Atkinson \& Hughes, 2008; Bradley, Lago-Peñas, Rey \& Gómez-Díaz, 2013). En la actualidad los entrenadores dan una gran importancia al aspecto táctico (Slimani et al., 2016), considerando la condición física y el dominio del balón como soporte de esta área de rendimiento (Barreira, Garganta, Castellano \& Anguera, 2014).

La evolución de los sistemas de juego junto con las modificaciones relativas al reglamento, han provocado que los jugadores tengan que aumentar durante la competición el número de acciones que realizan a alta intensidad (Bush, Barnes, Archer, Hogg \& Bradley, 2015) y adquirir un gran dominio sobre el balón para conseguir mantener el control del juego el mayor tiempo posible (Bradley et al., 2013). Quizás por ello, y aunque se ha observado que la ejecución de la técnica deportiva mejora con la maduración del jugador (Gil, Gil, Ruiz, Irazusta \& Irazusta, 2007) el entrenamiento de acciones como el regate durante el proceso de formación debería establecerse como un requisito fundamental para el 
desarrollo del talento de los jugadores jóvenes (Huijgen, Elferink-Gemser, Post \& Visscher, 2009). El regate parece tener gran importancia en el juego (Reilly, Williams, Nevill \& Franks, 2000). Tener futbolistas con esta habilidad se plantea como un importante recurso al servicio de la eficacia del comportamiento colectivo, generador de gran incertidumbre y provocador de la desestructuración de los planteamientos colectivos defensivos del rival (Castellano, Perea \& Hernández-Mendo, 2008). La habilidad para desplazarse a alta velocidad y regatear a jugadores rivales podría ser considerada una de las herramientas más importantes a la hora de provocar comportamientos de riesgo para el equipo rival (Ali, 2011), y por ello los mejores jugadores se pueden distinguir por su capacidad para mantener acciones de alta intensidad sin perder la eficacia en los enfrentamientos 1 vs 1 (Malina et al., 2005; Vaeyens et al., 2006).

Aunque tradicionalmente la técnica se relacionó con el dominio de patrones universales de movimiento, el carácter dinámico y cambiante que caracteriza a deportes como el fútbol, aconseja dotarla de flexibilidad (Bloomfield, Polman, O’Donoghue \& McNaughton, 2007). Por esta razón lejos de provocar automatizaciones las acciones técnicas como el regate deben ser desarrolladas a partir de la aplicación de estímulos que refuercen su dimensión cognitiva (Aquino et al., 2016), construyendo contextos donde la ejecución se produzca directamente relacionada con la variabilidad (García, Menayo, SánchezSánchez, 2015). Para conseguirlo los juegos reducidos (JRs) han sido considerados como una herramienta ideal para mejorar aspectos del juego con gran especificidad (Ford, Yates \& Williams, 2010). Los JRs tienen la capacidad de replicar las demandas físicas, tácticas y técnicas asociadas a la competición (Little, 2009). En cuanto a las acciones técnicas habitualmente se ha sugerido que emplear tareas con menor número de jugadores es más eficaz para potenciar esta habilidad (Jones \& Drust, 2007; Katis \& Kellis, 2009). Sin embargo, esta circunstancia no ha podido ser refrendada en otros trabajos que no observaron diferencias en la frecuencia de pase, regate o conducción (Abrantes, Nunes, MaÇãs, Leite \& Sampaio, 2012), o de entradas, interceptaciones y regates (Febré, Ríos, Casamichana, Gómez, Ríos \& Tamayo, 2015) al reducir el número de jugadores.

Aunque el análisis de las tareas de entrenamiento y su influencia sobre la demanda técnica es importante, en fútbol base también es interesante analizar la influencia de la propia competición sobre los factores de rendimiento (Lapresa, Arana, Garzón, Egüen \& Amatria, 2010). En una comparativa en categoría sub8 entre la modalidad de fútbol 3 (F3) y fútbol 5 (F5), el número de acciones técnicas fue mayor en F3 (Lapresa et al., 2010). Amatria (2015) expone que en categoría sub-10 los jugadores de fútbol 7 (F7) realizaban una mayor cantidad de acciones técnicas y con un mayor éxito que los jugadores de la modalidad de fútbol 8 (F8). Estas diferencias pueden explicarse por el espacio de interacción individual (EII) existente en cada modalidad (Katis \& Kellis, 2009; Owen, Twist \& Ford, 2004). El Ell se relaciona con la superficie de juego teórica que corresponde a cada jugador (Casamichana \& Castellano, 2010). A menor Ell la dificultad de ejecutar acciones técnicas aumentará, lo que condicionará el tipo de habilidades ejecutadas y su eficacia (Sanchez-Sanchez et al.,2017). 
El objetivo de este trabajo fue analizar la influencia de las modalidades de juego aplicadas a la competición en categoría sub-12 sobre la acción técnica del regate. Como hipótesis hemos señalado que el número de regates y su eficiencia será mayor en las modalidades con menos número de jugadores.

\section{MÉTODO}

\subsection{MUESTRA}

La muestra objeto de estudio estuvo compuesta por 785 acciones de regate, registradas a partir del análisis de 18 partidos de categoría sub-12 correspondientes a cada modalidad de competición ( $F 7, n=6 ; F 8, n=6 ; F 11, n=6)$. Los partidos se seleccionaron en función de los siguientes criterios de inclusión: los partidos debían ser disputados por equipos con jugadores en último año de categoría sub-12; los partidos debían formar parte de la competición sub-12 de mayor categoría presente en cada Federación de referencia. Los partidos de F7 analizados estaban organizados por la Federación de Castilla y León, los de F8 por la Federación Asturiana de Fútbol y los de F11 por la Federación de Fútbol de Madrid. La realización del estudio contó con la aprobación de los departamentos técnicos de los clubes participantes. Antes de realizar las grabaciones de los partidos, los padres o tutores de los jugadores firmaron el correspondiente consentimiento informado donde se explicaba de manera breve pero detallada los objetivos, beneficios, derechos y obligaciones asociadas a su participación. El diseño se realizó respetando todo lo dispuesto en la Declaración de Helsinki.

\subsection{PROCEDIMIENTO}

El presente trabajo es un diseño observacional basado en la grabación de partidos de categoría sub-12, que se disputaron bajo modalidades de competición diferentes. La grabación de los partidos fue realizada en el propio campo de juego. Para ello se utilizó una cámara de vídeo (Panasonic HC-V700, Panasonic ${ }^{\circledR}$, Osaka, Japón) situada a unos 10 metros de la zona de juego y sobre una altura de unos 7 metros (Sanchez-Sanchez, Carretero, Assante, Casamichana \& Los Arcos, 2015). Una vez grabados y descargados los partidos, fueron analizados por un observador experto empleando el programa LINCE v 1.1. (Gabin, Camerino, Anguera \& Castañer, 2012). Previo a la recogida de datos, el observador realizó 5 sesiones de práctica/aprendizaje para familiarizarse con la herramienta, así como con la variable dependiente objeto de análisis. Posteriormente, la fiabilidad intra-observador fue comprobada comparando los datos de 2 sesiones de análisis separadas 15 días, empleando el mismo partido de F7 (Casamichana \& Castellano, 2009). Los resultados mostraron una alta fiabilidad con un porcentaje de acuerdo del $95 \%$.

Debido a la diversidad de situaciones y acciones que se pueden observar en el fútbol es necesario preparar herramientas ad hoc (Anguera \& Hernández-Mendo, 
2013), por lo que se construyó un instrumento de observación que incluía botoneras para el registro de las variables. Estas botoneras hacían referencia a las variables dependientes número de regates totales $(R T)$, regates ganados (RG) y perdidos (RP) realizados en cada partido de F7, F8 y F11 en diferentes zonas del campo (variable independiente). El regate se ha considerado como una acción técnica consistente en una finta más o menos compleja que se realiza ante la acometida de un jugador rival (González-Víllora, 2010). En nuestro trabajo, se consideró como RG aquel en que el jugador que tiene el balón desborda al adversario manteniendo la posesión. Como RP se consideró aquellas acciones en las que el jugador atacante no conseguía superar a su adversario y perdía la posesión del balón.

El registro de las variables dependientes se realizó en función de las distintas modalidades de competición utilizadas en categoría sub-12 en España. Estas modalidades presentan un reglamento propio, tal y como se describe en la Tabla 1 (IFAB, 2016).

Tabla 1. Reglamento de juego de las modalidades de competición analizadas.

\begin{tabular}{|c|c|c|c|}
\hline Norma & F7 & F8 & F11 \\
\hline Terreno de juego & \multicolumn{2}{|c|}{$50-65 \times 30-45 \mathrm{~m}}$. & $90-120 \times 45-90 \mathrm{~m}$. \\
\hline El balón & \multicolumn{2}{|c|}{$62-66 \mathrm{~cm}$. Talla 4.} & $68-70 \mathrm{~cm}$. Talla 5. \\
\hline Número de jugadores & $\begin{array}{c}7 \text { por equipo. } \\
14 \text { totales. }\end{array}$ & $\begin{array}{c}8 \text { por equipo. } \\
16 \text { totales. }\end{array}$ & $\begin{array}{c}11 \text { por equipo. } \\
22 \text { totales. }\end{array}$ \\
\hline Fuera de juego & $\begin{array}{c}\text { A partir de la línea de } 12 \text { metros en el } \\
\text { sentido del ataque. }\end{array}$ & $\begin{array}{c}\text { A partir de la línea de medio campo en } \\
\text { el sentido del ataque. }\end{array}$ \\
\hline Sustituciones & $\begin{array}{c}\text { Ilimitadas. Se puede salir y volver a entrar } \\
\text { cuantas veces se quiera. }\end{array}$ & $\begin{array}{c}5 \text { sustituciones. El jugador sustituido no } \\
\text { puede entrar más. }\end{array}$ \\
\hline Tamaño portería & \multicolumn{2}{|c|}{$6 \times 2 \mathrm{~m}$} & $7,32 \times 2,44 \mathrm{~m}$ \\
\hline
\end{tabular}

Nota: F7 = Fútbol 7; F8 = Fútbol 8; Fútbol $11=\mathrm{F} 11 ; \mathrm{m}=$ metros. $\mathrm{cm}=$ centímetros.

Además, se contabilizó el número de regates en función de la zona del campo (Castelo, 1999). Para ello se empleó un campograma que determinaba 3 subespacios verticales o pasillos (Figura 1A): pasillo lateral derecho (PLD; F7 y $\left.\mathrm{F} 8=12 \times 60=720 \mathrm{~m}^{2} ; \mathrm{F} 11=20 \times 100=2.000 \mathrm{~m}^{2}\right)$; pasillo central $(\mathrm{PC} ; \mathrm{F} 7 \mathrm{y}$ $\mathrm{F} 8=24 \times 60=1.440 \mathrm{~m}^{2} ; \mathrm{F} 11=40 \times 100=4.000 \mathrm{~m}^{2}$ ); pasillo lateral izquierdo (PLI; F7 y $\left.\mathrm{F} 8=12 \times 60=720 \mathrm{~m}^{2} ; \mathrm{F} 11=20 \times 100=2.000 \mathrm{~m}^{2}\right)$. Para el análisis de resultados se consideraron los regates realizados en PC y pasillo lateral (PLD+PLI).También se utilizó otro campograma para dividir el campo de juego en 4 espacios horizontales o sectores (Figura 1B): sector defensivo (SD: F7 y F8 $=15 \times 48=720$ $\mathrm{m}^{2} ; \mathrm{F} 11=25 \times 80=2.000 \mathrm{~m}^{2}$ ), sector medio campo defensivo (SMD; F7 y F8 = $\left.15 \times 48=720 \mathrm{~m}^{2} ; \mathrm{F} 11=25 \times 80=2.000 \mathrm{~m}^{2}\right)$, sector medio campo ofensivo (SMO; $F 7$ y F8 $=15 \times 48=720 \mathrm{~m}^{2} ; \mathrm{F} 11=25 \times 80=2.000 \mathrm{~m}^{2}$ ) y sector ofensivo (SO; F7 y $\left.\mathrm{F} 8=15 \times 48=720 \mathrm{~m}^{2} ; \mathrm{F} 11=25 \times 80=2.000 \mathrm{~m}^{2}\right)$. 
A)

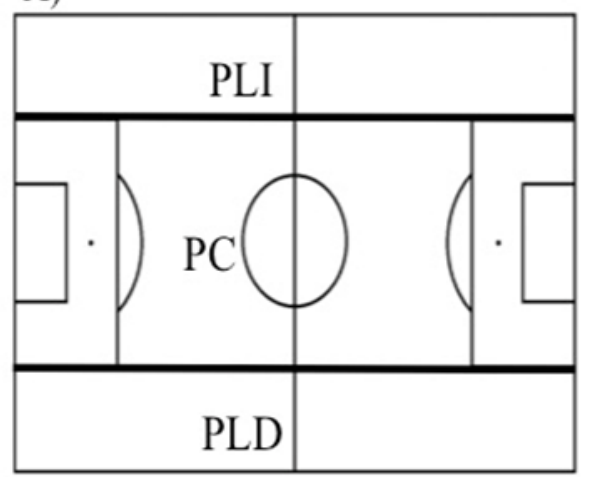

B)

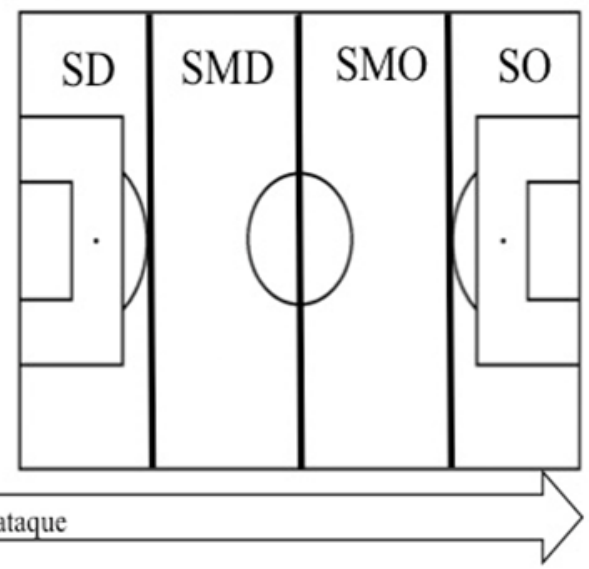

Figura 1. Campogramas que dividen el campo de juego en pasillos (A) y sectores (B). Nota: PLD = Pasillo Lateral Derecho; PC = Pasillo Central; PLI = Pasillo Lateral Izquierdo; SD =

Sector Defensivo. SMD = Sector Medio Campo Defensivo. SMO = Sector Medio Campo Ofensivo. SO = Sector Ofensivo.

\subsection{ANÁLISIS ESTADÍSTICO}

Los resultados se presentan como media \pm desviación estándar (DE). El estudio de las variables mostró una distribución normal de acuerdo a la prueba de Shapiro-Wilk. Para analizar la diferencia entre variables en función del tipo de modalidad de juego, se utilizó la prueba ANOVA de una vía, con post hoc de Tukey. Para comparar en cada modalidad el número de RT realizados en función del pasillo donde se producen (PLI+PLD vs PC), se utilizó la prueba $t$ de Student para muestras relacionadas. En todos los análisis se consideró diferencia significativa entre variables cuando $p<0,05$. Adicionalmente se valoró el tamaño del efecto (TE) a través de la prueba $d$ de Cohen (Cohen, 1988). El valor de $d$ fue $<0,1$ (muy pequeño), $0,1 \mathrm{a}<0,2$ (pequeño), 0,2 a $<0,5$ (moderado), $0,5 \mathrm{a}<$ 0,8 (grande) $y \geq 0,8$ (muy grande). El programa SPSS para Windows v. 20.0 (SPSS, Inc., Chicago IL.) fue utilizado para realizar el análisis estadístico.

\section{RESULTADOS}

No existían diferencias significativas en los RT en función de la modalidad de competición (Figura 2). Sin embargo TE mostró una diferencia muy grande en F11 vs $F 7(T E=1,21)$ y $F 8$ vs $F 7(T E=1,17)$. En $F 11$ el número de $R G$ fue significativamente mayor $(p<0,05)$ que en $F 7$. No se observaron diferencias en los RP entre modelos de competición, obteniéndose en todas las modalidades valores menores que en $R G(R P<R G: p<0,05$ en $F 7 ; p<0,01$ en $F 8$ y $F 11)$. 


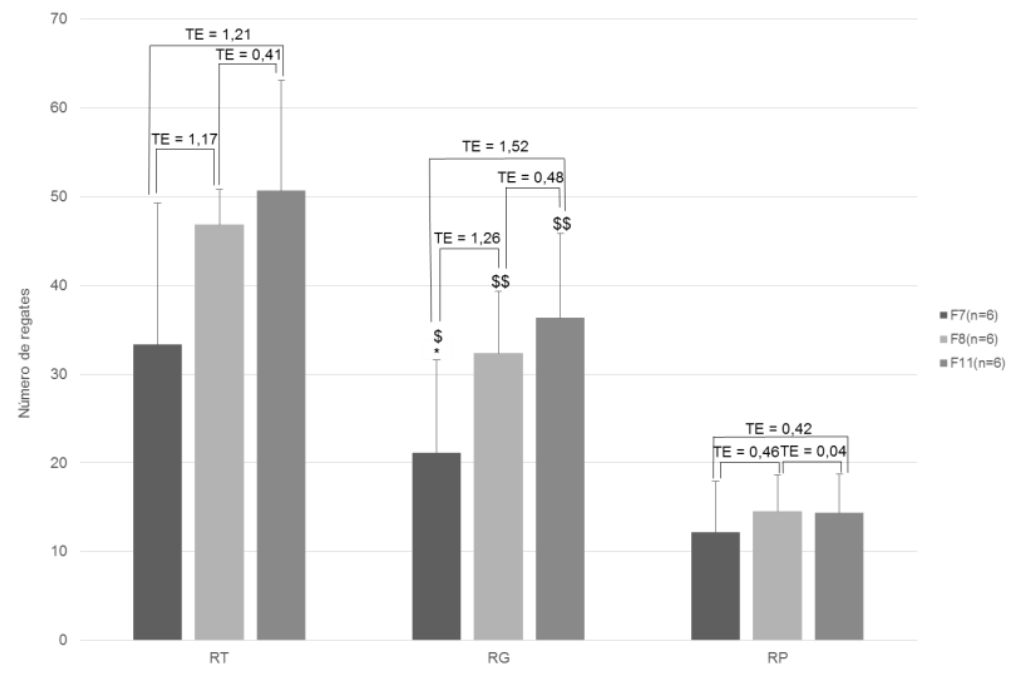

Figura 2. Número de regates realizados en función de la modalidad de competición. Nota: $T E=$ Tamaño del Efecto; $n=$ número de partidos analizados; $F 7=$ Fútbol 7; F8 = Fútbol 8; F11 = Fútbol 11; RT = Regates Totales; $R G=$ Regates Ganados; RP = Regates Perdidos. ${ }^{*}=$ Indica diferencias significativas con $\mathrm{F} 11(p<0,05) ;{ }^{\$} \mathrm{y} \$ \$$ = Indica diferencias significativas con $R P(p<0,05$ y $p<0,01$, respectivamente).

Según la Figura 3 la variable RG fue mayor que la RP tanto en $F 7(p<0,05)$ como en F8 y $F 11(p<0,01)$. Por otra parte, los RT y RG realizados en PLI+PLD en F11 fueron significativamente mayores $(p<0,05)$ que en $F 7$. En F8 la variable RT fue mayor $(p<0,05)$ que en F7. Con independencia de la modalidad de competición, en PLI+PLD se produjeron más RG que RP ( $p<0,05$ en F7 y F8; $p<0,01$ en F11). El análisis intramodalidad reflejó que en F8 y F11 la variable RT era significativamente mayor $(p<0,01)$ en PLD+PLI que en PC

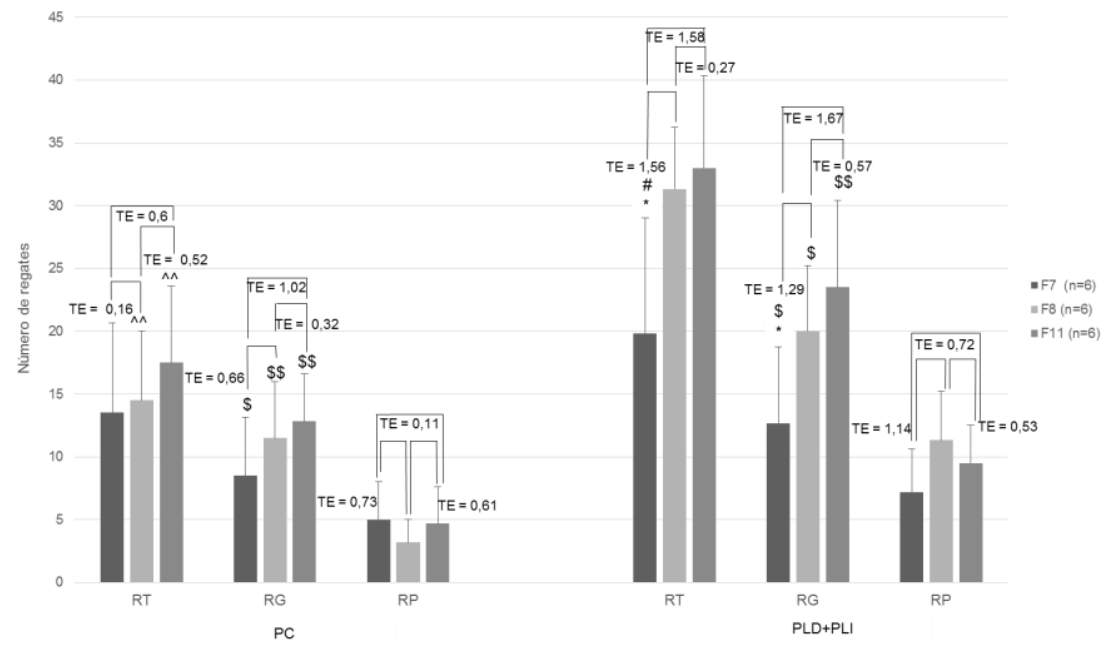

Figura 3. Número de regates realizados por pasillos en cada modalidad de competición. Nota: $T E=$ Tamaño del Efecto; $n=$ número de partidos analizados; $F 7=$ Fútbol 7; F8 = Fútbol 8; Fútbol $11=$ F11; RT = Regates Totales; $R G$ = Regates Ganados; RP = Regates Perdidos; $\mathrm{PC}=$ Pasillo Central; PLD + PLI = Pasillo Lateral Derecho más Pasillo Lateral Izquierdo. * ${ }^{*}$ Indica diferencias significativas con $\mathrm{F} 11(\mathrm{p}<0,05) ; \#=$ Indica diferencias significativas con F8 $(p<0,05) .{ }^{\$} y^{\$ \$}=$ Indica diferencias significativas con RP $(p<0,05$ y $p<0,01$, respectivamente); ${ }^{\wedge}=$ Indica diferencias con PLD+PLI $(p<0,01)$. 
El análisis por sectores determinó que en SMO las variables RT y RG eran mayores $(p<0,05)$ en F11 que en F7 (Tabla 2). Aunque sin valores estadísticamente significativos, en SMD existía una diferencia muy grande entre F11 y F7 en $R T(T E=1,17 ; 13,67 \pm 2,80$ vs $7,67 \pm 6,68)$ y $R G(T E=1,51 ; 10,67 \pm 3,44$ vs $5,00 \pm 4,0)$. El análisis intramodalidad reflejó que en todas las modalidades RT era significativamente más pequeño $(p<0,01)$ en SD y SMS que en SMO. En F8 y F11 la variable RT era menor $(p<0,01)$ en SD que en SMD y en SO. En este último sector también se realizaban menos regates $(p<0,01)$ que en SMO.

Tabla 2. Número de regates realizados por sectores en cada modalidad de competición.

\begin{tabular}{|c|c|c|c|c|c|c|c|}
\hline & & F7 $(n=6)$ & F8 $(n=6)$ & $\mathrm{F} 11(\mathrm{n}=6)$ & $\mathrm{TE}^{\mathrm{a}}$ & $T^{T^{b}}$ & $\mathrm{TE}^{\mathrm{c}}$ \\
\hline \multirow{3}{*}{ SD } & RT & $0,83 \pm 1,17^{\$}$ & $0,83 \pm 1,17^{\$ \#^{\wedge}}$ & $0,17 \pm 0,41^{\$ \#^{\wedge}}$ & 0 & 0,62 & 0,62 \\
\hline & $R G$ & $0,83 \pm 1,17$ & $0,83 \pm 1,17$ & 0,00 & 0 & 1,01 & 1,01 \\
\hline & $\mathrm{RP}$ & 0,00 & 0,00 & $0,17 \pm 0,41$ & - & 0,58 & 0,58 \\
\hline \multirow{3}{*}{ SMD } & RT & $7,67 \pm 6,68^{\$}$ & $11,00 \pm 4,60^{\$}$ & $13,67 \pm 2,80^{\$}$ & 0,58 & 1,17 & 0,7 \\
\hline & $R G$ & $5,00 \pm 4,05$ & $8,00 \pm 4,47$ & $10,67 \pm 3,44$ & 0,7 & 1,51 & 0,67 \\
\hline & RP & $2,67 \pm 2,94$ & $3,00 \pm 2,10$ & $3,00 \pm 1,26$ & 0,13 & 0,15 & 0 \\
\hline \multirow{3}{*}{ SMO } & RT & $17,33 \pm 9,35^{*}$ & $27,17 \pm 3,06^{\wedge}$ & $28,83 \pm 8,68^{\wedge}$ & 1,41 & 1,27 & 0,26 \\
\hline & $R G$ & $11,17 \pm 5,60^{*}$ & $18,50 \pm 2,81$ & $20,50 \pm 5,89$ & 1,66 & 1,62 & 0,43 \\
\hline & $\mathrm{RP}$ & $6,17 \pm 4,26$ & $8,67 \pm 3,39$ & $8,33 \pm 4,23$ & 0,65 & 0,51 & 0,09 \\
\hline \multirow{3}{*}{ SO } & RT & $8,00 \pm 5,90$ & $7,83 \pm 1,83$ & $7,83 \pm 1,83$ & 0,04 & 0,03 & 0 \\
\hline & $R G$ & $5,33 \pm 4,63$ & $5,17 \pm 2,79$ & $5,00 \pm 3,35$ & 0,04 & 0,08 & 0,05 \\
\hline & RP & $2,67 \pm 1,86$ & $2,67 \pm 1,86$ & $2,83 \pm 1,72$ & 0 & 0,09 & 0,1 \\
\hline
\end{tabular}

Nota: $\mathrm{n}=$ número de partidos analizados; $\mathrm{F} 7=$ Fútbol $7 ; \mathrm{F} 8=$ Fútbol 8; Fútbol $11=\mathrm{F} 11 ; \mathrm{TE}^{\mathrm{a}}=$ Tamaño del efecto $F 7$ vs F8; $T^{\mathrm{b}}=$ Tamaño del efecto $F 7$ vs $F 11 ; \mathrm{TE}^{\mathrm{c}}=$ Tamaño del efecto $\mathrm{F} 8$ vs F11; RT = Regates Totales; RG = Regates Ganados; RP = Regates Perdidos; SD = Sector Defensivo; SMD = Sector Medio Campo Defensivo; SMO = Sector Medio Campo Ofensivo; SO $=$ Sector Ofensivo; ${ }^{*}=$ Indica diferencias significativas con F11 $(p<0,05) ;{ }^{*}=$ Indica diferencias significativas con SMD $(p<0,01) ; \$=$ Indica diferencias significativas con SMO $(p<0,01)$; $\wedge^{\wedge}=$ Indica diferencias significativas con SO $(p<0,01)$.

\section{DISCUSIÓN}

El objetivo de este trabajo fue analizar la influencia de las modalidades de juego aplicadas a la competición en categoría sub-12, sobre la acción técnica del regate. Los resultados mostraron un incremento de RT en F8 respecto a F7. Una mayor densidad de jugadores en F8 respecto a F7, incrementa la dificultad para encontrar espacios y puede influir en el tipo de intervención del jugador (Lapresa, Álvarez, Anguera, Arana \& Garzón, 2015). Algunos trabajos han observado que cuánto más pequeño es el Ell mayor número de regates se realizaban (Febré et al., 2015; Katis \& Kellis, 2009; Owen, Twist \& Ford, 2004; Platt, Maxwell, Horn, Williams \& Reilly, 2001). El jugador puede tener dificultad para encontrar líneas de pase, teniendo que recurrir a la realización de acciones técnicas individuales como el regate (Sanchez-Sanchez et al., 2017). 
Por otra parte, en nuestro estudio también se muestra que el Ell mayor de F11 propicia más acciones de regate, apreciándose una diferencia muy grande con F7. Esto es contrario a lo obtenido en estudios previos que registraron más acciones de regate en las modalidades con menos Ell (Capranica, Tessitore, Guidetti \& Figura, 2001; Arana, Lapresa, Garzón, \& Álvarez, 2004). Un mayor Ell en F11 (300 $\mathrm{m}^{2} /$ jugador), respecto a F7 (183,5 m²/jugador) y F8 (157 m²/jugador) (IFAB, 2016) puede hacer que la organización colectiva se modifique. En F11 los jugadores pueden encontrarse dentro del terreno de juego muy separados, existiendo menos compañeros y adversarios alrededor del poseedor del balón. Esto fomentaría la aparición de una acción individual como el regate, ya que por una parte el juego colectivo ofensivo a través de pases se reduce (SanchezSanchez et al., 2017). Además nuestros resultados muestran que esta acción es altamente eficaz, debido a las diferencias en RG entre F11 y F7. Las dimensiones del terreno de juego en F11 dificultan al equipo defensor para realizar ayudas defensivas, lo que podría favorecer el éxito en la acción.

Independientemente de la modalidad practicada, en esta categoría los jugadores realizan un mayor número de $\mathrm{RG}$ que de $\mathrm{RP}$. Sin embargo, trabajos realizados con jugadores sub-8 y sub-10 en la modalidad F5 y F7 (Lapresa, Amatria, Egüen, Arana \& Garzón, 2008) o en JRs 2 vs 2 (Vera, Pino, Romero \& Moreno, 2007), obtuvieron lo contrario. Para poder realizar comparaciones entre los diferentes estudios, sería necesario tener en cuenta el nivel técnico de los jugadores.

Respecto a los datos obtenidos por pasillos, observamos que en PLD+PLI el número de RT en F11 y F8 es mayor que en F7. Además en F8 y F11 la variable RT era mayor en PLD+PLI que en PC. Respecto a los sectores, en el SMD y SMO la variable RT es mayor en F11 y F8 que en F7. En todas las modalidades SD era el sector donde menos regates se desarrollaban. No existen estudios que analicen la influencia de las zonas del campo sobre la ejecución de acciones técnicas como el regate, pero nuestros resultados podrían explicarse por el diferente dominio del espacio en profundidad y amplitud que los jugadores de estas edades realizan del terreno de juego (Lapresa, Arana, Ugarte \& Garzon, 2009). Del registro de las acciones de regate en F8 y F11 se desprende un cierto comportamiento técnico-táctico básico que no se observa en F7. La existencia de más acciones de regate en las zonas laterales del campo dentro de estas modalidades, puede tener como objetivo el ganar profundidad en el juego. Aunque no ha sido analizado en nuestro estudio, podríamos pensar que las características del F7 facilitarían la consecución de este comportamiento táctico no por el regate, sino por acciones colectivas. Por otra parte, en todas las modalidades observamos que el regate se realiza en sectores lejanos a la propia portería (SO y SMO), subrayando el riesgo que tiene esta acción técnica y la inteligencia táctica por parte del jugador, quien no sólo ejecuta correctamente a la vista de la relación entre RG y RP, sino que también es capaz de decidir el mejor contexto para la puesta en escena de las acciones con balón. 


\section{CONCLUSIONES}

La dimensión del espacio de juego donde se desarrolla la competición en categoría sub-12 determina el número de regates realizados. Con independencia de la modalidad, siempre se han observado más regates ganados que perdidos. En todas las modalidades el menor número de regates se realiza en el sector defensivo.

\section{REFERENCIAS BIBLIOGRÁFICAS}

Abrantes, C., Nunes, M., MaÇãs, V., Leite, N., \& Sampaio, J. (2012). Effects of the number of players and game type constraints on heart rate, rating of perceived exertion, and technical actions of small-sided soccer games. The Journal of Strength \& Conditioning Research, 26(4), 976-981. https://doi.org/10.1519/JSC.0b013e31822dd398

Ali, A. (2011). Measuring soccer skill performance: A review. Scandinavian Journal of Medicine and Science in Sports, 21(2), 170-183. https://doi.org/10.1111/j.1600-0838.2010.01256.x

Amatria, M. (2015). Análisis observacional del desempeño técnico-táctico en la fase ofensiva de las modalidades de fútbol sala, fútbol 7 y fútbol 8 , en categoría benjamín. (Tesis Doctoral Inédita). Universidad de la Rioja.

Anguera, M. T., \& Hernández-Mendo, A. (2013). Observational methodology in sport sciences. Journal of Sports Science, 9(3), 135-160.

Aquino, R., Cruz, L., Palucci, L., Oliveira, L., Alves, G., Pereira, P., \& Puggina, E. (2016). Periodization Training Focused on Technical-Tactical Ability in Young Soccer Players Positively Affects Biochemical Markers and Game Performance. Journal of Strength and Conditioning Research, 30(10), 2723-2732. https://doi.org/10.1519/JSC.0000000000001381

Arana, J., Lapresa, D., Garzón, B., \& Álvarez, A. (2004). La alternativa del fútbol 9 para el primer año de la categoría infantil. Universidad de La Rioja, Logroño.

Barreira, D., Garganta, J., Castellano, J., \& Anguera, M. T. (2014). Evolución del ataque en el fútbol de élite entre 1982 y 2010: Aplicación del análisis secuencial de retardos. Revista de Psicologia Del Deporte, 23,139-146.

Bloomfield, J., Polman, R., O'Donoghue, P., \& McNaughton, L. (2007). Effective speed and agility conditioning methodology for random intermittent dynamic type sports. Journal of Strength \& Conditioning Research, 21(4), 10931100. https://doi.org/10.1519/00124278-200711000-00020

Bradley, P. S., Lago-Peñas, C., Rey, E., \& Gomez-Diaz, A. (2013). The effect of high and low percentage ball possession on physical and technical profiles in English FA Premier League soccer matches. Journal of Sports Sciences, 31(12), 1261-1270. https://doi.org/10.1080/02640414.2013.786185

Bush, M., Barnes, C., Archer, D. T., Hogg, B., \& Bradley, P. S. (2015). Evolution of match performance parameters for various playing positions in the English Premier League. Human Movement Science, 39, 1-11. https://doi.org/10.1016/j.humov.2014.10.003 
Capranica, L., Tessitore, A., Guidetti, L., \& Figura, F. (2001). Heart rate and match analysis in pre-pubescent soccer players. Journal of Sports Sciences, 19(6), 379-384. https://doi.org/10.1080/026404101300149339

Casamichana, D., \& Castellano, J. (2009). Análisis de los diferentes espacios individuales de interacción y los efectos en las conductas motrices de los jugadores: aplicaciones al entrenamiento en futbol. Motricidad. European Journal of Human Movement, 23, 143-167.

Casamichana, D., \& Castellano, J. (2010). Time-motion, heart rate, perceptual and motor behaviour demands in small-sides soccer games: Effects of pitch size. Journal of Sports Sciences, 28(14), 1615-1623. https://doi.org/10.1080/02640414.2010.521168

Castelo, J. (1999). Fútbol: Estructura y dinámica del juego. Barcelona: Inde.

Castellano, J., Perea, A., \& Hernández-Mendo, A. (2008). Análisis de la evolución del fútbol a lo largo de los mundiales. Psicothema, 20(4), 928932.

Cohen, J. (1988). Statistical power analysis for the behavioral sciences. Hillsdale, NJ: Lawrence Earlbaum Associates.

Febré, R., Ríos, L. J. C., Casamichana, D., Gómez, D. C., Ríos, I. C., \& Tamayo, I. M. (2015). Influencia de la densidad de jugadores sobre la frecuencia cardíaca y respuestas técnicas en jóvenes jugadores de fútbol. RICYDE: Revista Internacional de Ciencias Del Deporte, 11(41), 226-244. https://doi.org/10.5232/ricyde2015.04002

Ford, P., Yates, I. \& Williams, A. M. (2010). An analysis of practice activities and instructional behaviours used by youth soccer coaches during practice: Exploring the link between science and application. Journal of Sports Sciences, 28(5), 483-495. https://doi.org/10.1080/02640410903582750

Gabin, B., Camerino, O., Anguera, M. T. \& Castañer, M. (2012). Lince: multiplatform sport analysis software. Procedia-Social and Behavioral Sciences, 46, 4692-4694. https://doi.org/10.1016/j.sbspro.2012.06.320

García, J.A., Menayo, R. \& Sanchez-Sanchez, J. (2015) Efectos de la práctica variable sobre el golpeo a portería en fútbol. Revista Internacional de Medicina y Ciencias de la Actividad Física y el Deporte, 15(60), 438-445. https://doi.org/10.15366/rimcafd2015.60.004

Gil, S. M., Gil, J., Ruiz, F., Irazusta, A., \& Irazusta, J. (2007). Physiological and anthropometric characteristics of young soccer players according to their playing position: relevance for the selection process. Journal of Strength and Conditioning Research, 21(2), 438-445. https://doi.org/10.1519/00124278-200705000-00026

González-Víllora, S. (2010). Estudio de las etapas de formación del joven deportista desde el desarrollo de la capacidad táctica. Aplicación al fútbol. Tesis Doctoral publicada. Cambridge (Reino Unido). Editorial Proquest.

Huijgen, B. C. H., Elferink-Gemser, M. T., Post, W. J., \& Visscher, C. (2009). Soccer skill development in professionals. International Journal of Sports Medicine, 30(8), 585-591. https://doi.org/10.1055/s-0029-1202354

IFAB. (2016). Reglas del Juego 2016/2017. Zúrich: FIFA.

Jones, S., \& Drust, B. (2007). Physiological and technical demands of $4 \mathrm{v} 4$ and 8 v 8 games in elite youth soccer players. Kinesiology, 39(2), 150-156. 
Katis, A. \& Kellis, E. (2009). Effects of small-sided games on physical conditioning and performance in young soccer players. Journal of sports science and medicine, 8(3), 374-380.

Lapresa, D., Amatria, M., Egüen, R., Arana, J. \& Garzón, B. (2008). Análisis descriptivo y secuencial de la fase ofensiva del fútbol 5 en la categoría prebejamin. Cultura, Ciencia y Deporte, 3(8), 107-116. https://doi.org/10.12800/280

Lapresa, D., Arana, J., Ugarte, J. y Garzón, B. (2009). Análisis comparativo de la acción ofensiva en F-7 y F-8, en la categoría alevín. Retos. Nuevas Tendencias En Educación Física, Deporte Y Recreación, 2041(16), 99-103.

Lapresa, D., Arana, J., Garzón, B., Egüen, R., \& Amatria, M. (2010). Adaptando la competición en la iniciación al fútbol: estudio comparativo de las modalidades de fútbol 3 y fútbol 5 en categoría prebenjamín. Apunts. Educación Física y Deportes, 101, 43-56.

Lapresa, D., Álvarez, I., Anguera, M. T., Arana, J., \& Garzón, B. (2015). Comparative analysis of the use of space in 7-a-side and 8-a-side soccer: How to determine minimum sample size in observational methodology. Motricidade, 11(4), 92-103. https://doi.org/10.6063/motricidade.4138

Lee, M. J., Whitehead, J., \& Ntoumanis, N. (2007). Development of the attitudes to moral decision-making in youth sport questionnaire (AMDYSQ). Psychol, 369-392. https://doi.org/10.1016/i.psychsport.2006.12.002

Little, T. (2009). Optimizing the use of soccer drills for physiological development. Strength \& Conditioning Journal, 31(3), 67-74. https://doi.org/10.1519/SSC.0b013e181a5910d

Malina, R. M., Cumming, S. P., Kontos, A. P., Eisenmann, J. C., Ribeiro, B., \& Aroso, J. (2005). Maturity-associated variation in sport-specific skills of youth soccer players aged 13-15 years. Journal of Sports Sciences, 23(5), 515522. https://doi.org/10.1080/02640410410001729928

Nevill, A., Atkinson, G., \& Hughes, M. (2008). Twenty-five years of sport performance research in the Journal of Sports Sciences. Journal of Sports Sciences, 26(4), 413-426. https://doi.org/10.1080/02640410701714589

Owen, A., Twist, C. \& Ford, P. (2004). Small-sided games: the physiological and technical effect of altering pitch size and player numbers. Insight, 7(2), 5053.

Platt, D., Maxwell, A., Horn, R., Williams, M. \& Reilly, T. (2001). Physiological and technical analysis of $3 \vee 3$ and $5 \vee 5$ youth football matches. Insight, 4(4), 2324.

Reilly, T., Bangsbo, J., \& Franks, A. (2000). Anthropometric and physiological predispositions for elite soccer. Journal of Sports Sciences, 18(9), 669-683. https://doi.org/10.1080/02640410050120050

Reilly, T., Williams, A. M., Nevill, A., \& Franks, A. (2000). A multidisciplinary approach to talent identification in soccer. Journal of Sports Sciences, 18, 695-702. https://doi.org/10.1080/02640410050120078

Sanchez-Sanchez, J., Carretero, M., Assante, G., Casamichana, D., \& Los Arcos, A. (2015). Efectos del marcaje al hombre sobre la frecuencia cardíaca, el esfuerzo percibido y la demanda tecnico-tactica en jóvenes 
jugadores de fútbol. RICYDE: Revista Internacional de Ciencias Del Deporte, 12(44), 90-106. https://doi.org/10.5232/ricyde2016.04401

Sanchez-Sanchez, J., Hernandez, D., Casamichana, D., Martinez, C., RamírezCampillo, R., \& Sampaio, J. (2017). Heart rate, technical performance and session-RPE in elite youth soccer small-sided games played with wildcard player. Journal of Strength \& Conditioning Research, 31(10), 2678-2685. https://doi.org/10.1519/JSC.0000000000001736

Slimani, M., Bragazzi, N. L., Tod, D., Dellal, A., Hue, O., Cheour, ... \& Chamari, K. (2016). Do cognitive training strategies improve motor and positive psychological skills development in soccer players? Insights from a systematic review. Journal of Sports Sciences, 34(24), 2338-2349. https://doi.org/10.1080/02640414.2016.1254809

Vaeyens, R., Malina, R. M., Janssens, M., Van Renterghem, B., Bourgois, J., Vrijens, J., \& Philippaerts, R. (2006). A multidisciplinary selection model for youth soccer: the Ghent Youth Soccer Project. British Journal of Sports Medicine, 40(11), 928-934. https://doi.org/10.1136/bjsm.2006.029652

Vera, G., Pino, J., Romero, C., y Moreno, M. I. (2007). Propuesta de valoración técnico-táctica mediante una situación de juego colectivo básico en el fútbol de iniciación. Retos. Nuevas Tendencias en Educación Física, Deporte y Recreación, 12, 29-35.

Referencias totales / Total references: 47 (100\%)

Referencias propias de la revista / Journal's own references: 1 (2,13\%)

Rev.int.med.cienc.act.fís.deporte - vol. 19 - número 75 - ISSN: 1577-0354 